\title{
Transculturación, transmodernidad y traducción. Una mirada latinoamericana sobre la Europa del siglo XXI
}

Transculturation, Transmodernity and Translation.

21 st Century Europe viewed from Latin America

Transculturação, transmodernidade e tradução.

A Europa do século XXI vista da América Latina

\section{Christiane Stallaert}

\section{UNIVERSITEIT ANTWERPEN, BÉLGICA}

Doctora en Antropología Social y Cultural por la Catholic University of

Leuven (Bélgica). Autora de $\mathcal{N i}$ una gota de sangre impura. La España

inquisitorial y la Alemania nazi cara a cara (Galaxia Gutenberg/

Círculo de Lectores, 2006), Perpetuum mobile. Entre la balcanización y

la aldea global (Anthropos, 2004) y Etnogénesis y etnicidad en España.

Una aproximación histórico-antropológica al casticismo (Anthropos,

1998). Correo electrónico: christiane.stallaert@uantwerpen.be

\footnotetext{
Artículo de reflexión

Documento accesible en línea desde la siguiente dirección: http://revistas.javeriana.edu.co

Ponencia leída en el Simposio "La producción, traducción y apropiación

transregional de saberes: actores, instituciones y discursos", organizado por el Ibero-

Amerikanisches Institut y llevado a cabo el 10 y 11 de octubre de 2014.
} 


\section{Resumen}

Las grandes metrópolis europeas están inmersas en procesos de transculturación cultural y social sin precedentes. En este contexto, proponemos invertir la tradicional mirada eurocéntrica como estrategia epistemológica y estudiar las nuevas realidades sociales y culturales europeas desde América Latina. Destacamos el interés del enfoque trans para entender la compleja realidad antropológica de un mundo globalizado. Nuestras reflexiones se centran en tres conceptos: transculturación, transmodernidad y traducción. En cuanto a esta última, proponemos repensar las teorías eurocéntricas, privilegiando el paradigma perspectivista, que permite deconstruir la dicotomía jerarquizada original/copia que define como sincretismos (impuros, inferiores) los productos de los procesos de transculturación. A modo de ejemplo nos referiremos en nuestro análisis a la ciudad de Bruselas, sede de la Unión Europea y capital del Estado belga.

Palabras clave: globalización; transculturación; transmodernidad; traducción; Europa

\section{Abstract}

The great European metropolises are immersed in unprecedented processes of cultural and social transculturation. In this context, we propose to reverse the traditional Eurocentric view as an epistemological strategy and to study the new European social and cultural realities from Latin America. We emphasize the interest of the trans-approach to understand the complex anthropological reality of a globalized world. Our reflections focus on three concepts: transculturation, trans-modernity, and translation. Regarding the latter, we propose to rethink the Eurocentric theories, favoring instead the perspectivist paradigm, which allows for the deconstruction of the hierarchical dichotomy original/copy that defines as an impure, inferior syncretism the products of the transculturation processes. By way of example, we will refer in our analysis to Brussels, seat of the European Union and capital of the Belgian State.

Keywords: globalization; transculturation; transmodernity; translation; Europe

\section{Resumo}

As grandes metrópoles europeias estão imersas em processos de transculturação cultural e social sem precedentes. Neste contexto, propomos inverter o tradicional olhar eurocêntrico como estratégia epistemológica e estudar as novas realidades sociais e culturais europeias desde América Latina. Enfatizamos o interesse da abordagem trans para compreender a complexa realidade antropológica de um mundo globalizado. As nossas reflexões estão focadas em três conceitos: transculturação, transmodernidade e tradução. No que diz respeito desta última, propomos repensar as teorias eurocêntricas, privilegiando o paradigma perspectivista, que permite desconstruir a dicotomia hierarquizada original/copia definindo como sincretismos (impuros, inferiores) os produtos dos processos de transculturação. A manera de exemplo, referirmo-nos na nossa análise à cidade de Bruxelas, sede da União Europeia e capital do Estado belga.

Palavras-chave: globalização; transculturação; transmodernidade; tradução; Europa

RECIBIDO: 22 DE ENERO DE 2016. ACEPTADO: 27 DE MARZO DE 2016. DISPONIBLE EN LÍNEA: 26 DE JUNIO DE 2017

Cómo citar este artículo:

Stallaert, Christiane. "Transculturación, transmodernidad y traducción. Una mirada latinoamericana sobre la Europa del siglo XXI". Cuadernos de Literatura 21.41 (2017): 131-152. https://doi.org/10.11144/Javeriana.cl21-41.tttm 


\section{Introducción}

Frente al lugar común de considerar a Latinoamérica como "el otro Occidente" y analizar el continente bajo el prisma eurocéntrico de un proceso de "occidentalización", Centeno y López-Alves, en The Other Mirror. Grand Theory through the Lens of Latin America (2000), proponen invertir la mirada para repensar críticamente las grandes teorías de ciencias sociales desde la realidad latinoamericana. Señalan que el interés del ejercicio rebasa lo estrictamente académico, ya que la nueva visión del mundo que esta mirada permite descubrir, puede influir en cómo se interactúa con él.

Con la sociedad europea del siglo XXI como telón de fondo, proponemos invertir el tradicional paradigma de la dependencia académica para empezar a repensar Europa desde Latinoamérica. Construimos nuestra propuesta sobre la interrelación que hay entre los conceptos de transculturación, transmodernidad y traducción. Las experiencias históricas con procesos de contacto cultural y mestizaje, así como su problemático encaje en el modelo político del Estado-nación, confieren a Latinoamérica un lugar central a la hora de estudiar los retos a los que se enfrenta la Europa de hoy. La enorme diversidad cultural de las grandes ciudades europeas, que surgió como consecuencia de las migraciones internacionales y la globalización, hace patente la discrepancia existente entre el histórico proyecto social y político común y la realidad sociodemográfica actual. Para las ciencias sociales el desafío es cómo estudiar, en tiempos de globalización, realidades culturales europeas que oscilan entre una creciente segregación y una hibridación, y parte de cuyas poblaciones no se sabe muy bien si están entrando o saliendo de la modernidad. A modo de ejemplo nos referiremos en nuestro análisis a la ciudad de Bruselas, sede de la Unión Europea y capital del Estado belga.

\section{Trans como (nuevo) paradigma de análisis}

Lo que a primera vista une los tres conceptos de transculturación, transmodernidad y traducción (translation) es el prefijo "trans". Este prefijo se impuso en la última década como un cambio de paradigma, aportando nuevo oxígeno a las ciencias humanas/sociales una vez quedó agotado el paradigma "post". Las convocatorias de congresos o títulos de revistas internacionales recientes parecen un festival de lo "trans": transculturalidad, transnacionalismo, transmigración, translingualismo, incluso en combinaciones creativas como "transcultural translations" o "transgressive translations". ${ }^{1}$ El cambio de paradigma fue mediado por el enfo-

$1 \quad$ Véanse, entre numerosos ejemplos, el congreso "Transcultural Identity Constructions in a Changing World", celebrado en Dalarna University, Suecia, del 2 al 4 de abril de 2014, o también, "Representing, (De)Constructing and Translating Borderlands", celebrado en Krasnogruda, Polonia, el 29 y 30 de mayo de 2014. 
que "fronterizo", que a pesar de la impronta chicana (y por tanto latinoamericana) se institucionalizaría con un nombre en inglés: Border Studies. La secuencia lógica que lleva desde la noción de frontera (border) - separación entre dos realidades distintas, pensadas como puras y homogéneas-, hacia el enfoque "trans", queda ilustrada en la obra de José David Saldívar a cuyo libro Border Matters, publicado en 1997, le sucede Trans-Americanity en 2011.

En el paso de esta evolución, el concepto de traducción (translation) ocupó y sigue ocupando un papel central. La traducción - por el movimiento que el término sugiere (derivado del latín traducere, trasladar)—, funciona como metáfora de la transgresión de la frontera; del movimiento capaz de unir - y contaminar - las realidades que la frontera pretende separar. Translation está asociado con crossing (cultural) borders; asociación que explica la aplicación del término a la antropología, ya sea como caracterización del trabajo del antropólogo - la antropología como traducción cultural (Asad) o como definición misma del concepto de cultura como transnational and translational, en palabras de Homi Bhabha. Esta última definición implica una visión dinámica que rompe con la visión esencialista en donde la cultura se asociaba de forma estática con el grupo nacional (moderno) o étnico (premoderno) (Bhabha). El giro "translacional" es la respuesta de los antropólogos a los desafíos planteados por un mundo globalizado, de flujos discontinuos que escapan a la práctica habitual de la antropología "situada"; el trabajo de campo en un lugar fijo. En palabras de George Marcus, la etnografía multisituada que requiere la nueva realidad social, significa para el antropólogo un mayor desafío de traducción (Marcus).

Aunque a mediados de los 9o, George Marcus estimaba que la antropología aún carecía de conceptos teóricos o de un modelo descriptivo que permitiera estudiar las nuevas culturas globalizadas (Marcus 86), la inversión de la mirada eurocéntrica, que se elabora desde la realidad latinoamericana, permite contradecir esta afirmación. Si el paradigma "trans" se ha contemplado como una solución a las nuevas necesidades conceptuales de la realidad global, vistos desde Latinoamérica ni el paradigma ni la propia realidad global pueden ser tildados de novedosos. En los estudios de migraciones, por ejemplo, fenómenos estudiados hoy bajo el denominador del "transnacionalismo" (redes, transporte, comunicación, remesas, bifocalidad, etc.) ya se encuentran documentados en las Cartas privadas de migrantes a Indias, 1540-1616, editadas por Enrique Otte (1996), así como el concepto de "transmigración" y una perspectiva profundamente "transcultural", que se pueden encontrar en la Historia Natural y Moral de las Indias, de José de Acosta. A diferencia del modelo occidental que hasta 
después de la Segunda Guerra Mundial se inspiraba en el ideal de lo puro y homogéneo, el pensamiento latinoamericano privilegiaba lo "trans" como clave de entendimiento de los procesos de transformación social y cultural en la región (Zalamea). Fue la propia realidad latinoamericana, pensada desde Europa como "otro Occidente" - es decir, como "un aprendizaje, un borrador o una copia de la cultura burguesa europea" (Fernández Retamar 9-11) - , la que estimuló a los pensadores latinoamericanos a desarrollar un marco conceptual que permitiera valorizar la "copia" frente al "original sagrado", retomando aquí la metáfora de Fernández Retamar. Uno de los conceptos más exitosos a este respecto fue el de "transculturación", acuñado por el antropólogo cubano Fernando Ortiz en 1939. Recordemos que, para Ortiz, la transculturación expresa un proceso complejo y dinámico bi- o multidireccional que abarca distintas fases de cambio social y cultural, desde la desculturación hasta la neoculturación.

La omnipresencia y el consenso que reina hoy en los estudios culturales occidentales sobre la utilidad de este concepto, oculta la lenta y dificultosa penetración que se desenvuelve hacia el centro de las ciencias humanas/sociales, del que estuvo prácticamente ausente hasta que Mary Louise Pratt lo recuperara para su obra Imperial Eyes. Travel Writing and Transculturation (1992), relegando a una mera nota a pie de página la paternidad de Ortiz. Desde su consagración por la academia estadounidense en 1992, cabe hablar de un auténtico boom del concepto, que, como destaca Diana Taylor (2012), en su periplo por las distintas tradiciones académicas y contextos culturales - principalmente las tradiciones francófona, hispano-lusa y anglófona-, pasó él mismo por un proceso de transculturación.

Otro concepto que irrumpió en la academia occidental es el de hibridación, teorizado en un contexto latinoamericano por el argentino Néstor García Canclini. En su libro Culturas híbridas: Estrategias para entrar y salir de la modernidad, cuya primera edición data de 1990, García Canclini define la hibridación como "procesos socioculturales en los que estructuras o prácticas discretas, que existían en forma separada, se combinan para generar nuevas estructuras, objetos y prácticas" (14). El autor enfatiza que cada hibridación es el resultado de hibridaciones anteriores, por lo que las estructuras o prácticas que subyacen al proceso no se han de considerar como puntos de origen puros u homogéneos. De ahí que sea preferible hablar de "ciclos de hibridación". Distinguiéndolo de una visión identitaria esencialista o fundamentalista, Canclini considera hibridación como un "término de traducción" (García Canclini 29) perteneciente a una red de conceptos afines tales como contradicción, mestizaje, sincretismo, creolización, fusión o transculturación; 
conceptos que evocan modalidades de mezcla. La finalidad de su esfuerzo de teorización es ofrecer instrumentos que nos ayuden a "traducir" el mundo. Como escribe Canclini, se trata de "seguir construyendo principios teóricos y procedimientos metodológicos que nos ayuden a volver este mundo más traducible, o sea convivible en medio de sus diferencias, y aceptar a la vez lo que cada uno gana y está perdiendo al hibridarse" (29-30).

\section{El turno de Europa}

Si la globalización se caracteriza por la aceleración e intensificación de los procesos de transculturación e hibridación, sus efectos son vividos en muchos países europeos como amenazantes para el fundamento político de la sociedad; el Estado-nación, que ha sido vinculado históricamente al imaginario de una comunidad homogénea y pura. Si los procesos de transculturación sociodemográfica de la "primera globalización" (la colonización de América a partir de 1492) tuvieron como escenario el Nuevo Mundo, en la actual "segunda globalización", las migraciones han convertido los procesos de transculturación e hibridación en realidades domésticas para el Viejo Mundo. En los estudios latinoamericanistas decoloniales, El primer nueva corónica y buen gobierno (1615) de Guaman Poma de Ayala ocupa un lugar icónico en su papel de alertador de lo que el autor percibe como "un mundo al revés"; es decir, la profunda transculturación social, cultural, política o religiosa, para la que "no hay remedio". Hoy le toca el turno a Europa, y en muchos países europeos reina la sensación de estarse viviendo un "mundo al revés", caracterizado por el camino irreversible hacia una nueva diversidad.

La presencia del islam se presenta como uno de los mayores desafíos sociales y culturales para la Europa del siglo XXI; realidad que afecta profundamente los grandes núcleos urbanos como Londres, Ámsterdam, Berlín, París o Bruselas. En cuanto a esta última ciudad, según datos de 2013, un 22,6\% de la población es musulmana, porción de la que más de la mitad es de origen marroquí. Ocho de los 19 distritos de Bruselas tienen una población musulmana que oscila entre el 20\% y el 43,7\%. ${ }^{2}$ Desde hace unos diez años, la lista de nacimientos en Bruselas va encabezada, con mucha ventaja, por el nombre de Mohamed, seguido en el top ten por otros nombres árabes como Ayoub, Mehdi, Bilal, Anas o Zakaria. Junto con la nacionalidad belga, la población

2 Datos elaborados por el sociólogo Jan Hertogen con fecha del 8 de octubre de 2014, KU Leuven. Ver: http://www.npdata.be/BuG/239-Moslims-2013/Moslims-2013.htm. Fecha de consulta: 07 de enero de 2015. 
de origen marroquí o turco conserva su nacionalidad de procedencia. Hoy un elevado porcentaje de la población de la ciudad tiene la doble, o inclusive, triple nacionalidad. Por otro lado, los datos del censo nacional de 2011 revelan que el $10 \%$ de la población de Bélgica no posee la nacionalidad belga y en Bruselas esta cifra asciende al 32,3\% de la población. ${ }^{3}$ En 2012, un 15,2\% de la población de Bélgica había nacido en el extranjero. ${ }^{4}$ Siendo Bruselas una ciudad oficialmente bilingüe (francés/neerlandés), hoy el francés es de hecho la lingua franca de una población bi- o multilingüe, y el inglés se ha convertido en la segunda lengua más hablada, por delante del neerlandés. El retroceso del neerlandés ha sido acompañado por un avance espectacular del árabe, lengua hablada por un 20\% de la población (frente al 10\% en el año 2000). Hoy, un 10\% de la población de la ciudad no habla ninguna de las tres lenguas mayoritarias (francés, inglés o neerlandés).

Si confrontamos estas cifras con la percepción de la realidad social, se averigua que en Bélgica existe una percepción general de que un 29\% de la población está compuesta por musulmanes (en realidad, un 6,5\%), cifra que coincide con la percepción que existe sobre el porcentaje de la población de origen extranjero, igualmente estimado en un $29 \%$ de la población (en realidad, un 10\%). ${ }^{5}$ Este dato es importante ya que demuestra cómo en la percepción de la realidad se establece una equiparación entre cambios demográficos e islamización.

Cabe añadir un dato más. Se ha calculado que en las pasadas elecciones de 2014, y sabiendo que en Bélgica existe la obligación de votar (a partir de los 18 años), alrededor de un cuarto de la población en edad de votar no pudo participar debido a que carecía de la nacionalidad belga, se abstuvo o emitió un voto nulo o en blanco. Para Bruselas esta cifra sube hasta el 47\%. Los politólogos advierten que el número de extranjeros en Bruselas seguirá aumentando en los próximos años, con lo cual en pocos años las elecciones dejarán de reflejar la voluntad política de una mayoría de la población. ${ }^{6}$ La realidad de Bruselas confirma las alertas de Stephen Castles sobre los efectos de las migraciones para el concepto de ciudadanía:

\footnotetext{
3 Véase el censo belga de 2011. www.census2011.be. Fecha de consulta: 07 de enero de 2015.

4 Datos de la OCDE. Ver "Une 'baisse des flux" vers la Belgique", Metro, 2 de diciembre de 2014.

5 Véase "Perils of perception. A fourteen country study". Ipsos MORI. http://goo.gl/vz4Zrj. Fecha de consulta: 07 de enero de 2015; "Hoeveel migranten wonen er nu echt in België?", Het Laatste Nieuws, 29 de octubre de 2014. http://goo.gl/kFKiKV. Fecha de consulta: 07 de enero de 2015.

6 Véase: "Slechts helft Brusselaars bracht stem uit". De Standaard. 18 de diciembre de 2014: 8-9. Impreso.
} 
Transnationalism will inevitably lead to a rapid rise in multiple citizenship -creating the phenomenon most feared by nationalists- the potentially divided loyalties of people with an instrumental rather than emotional attribute toward state membership. The growth of transnationalism may in the long run lead to a rethinking of the very contents of citizenship. (Citado en Koser 27)

Basándonos en las cifras anteriores, en términos de identidades colectivas el espacio europeo se puede representar hoy como una superficie compuesta por una parte de desiertos - amplias zonas en donde siguen predominando las identidades nacionales, regionales y/o locales históricas pensadas como homogéneas y arraigadas en el territorio-, y, por otra, de nodos: núcleos urbanos con una población crecientemente diversa, resultado de flujos migratorios internacionales. Conviene observar que esta última población, transplantada y territorialmente cortada de sus raíces identitarias de origen, no necesariamente se inscribe en un proyecto cosmopolita de acuerdo con una visión posmoderna de la identidad. $\mathrm{Al}$ contrario, en muchos casos se intenta mantener viva o incluso reforzar la identidad de origen, ya sea moderna (nacional) o premoderna (étnica/religiosa).

\section{Transmodernidad o el original suplantado por la copia}

Introducimos aquí el concepto de transmodernidad. Este concepto, a diferencia de las alternativas de surmodernidad o altermodernidad, y de acuerdo con el enfoque "trans", abarca identidades socioculturales modernas (nacionales), premodernas (étnicas) y posmodernas (híbridas o mestizas). Es decir que no rechaza la herencia de la modernidad sino que la abraza, aunque transformándola y trascendiéndola. La teoría de la transmodernidad, que nos parece útil para estudiar las realidades complejas de la Europa globalizada del siglo XXI, surgió en 1989 de forma simultánea en España y América Latina.

Para el filósofo argentino Enrique Dussel, la transmodernidad remite al diálogo intercultural transversal y simétrico de periferia a periferia (Sur-Sur) como "afirmación de la exterioridad despreciada", negada, del sistema-mundo (18-19). Mientras que Dussel concibe la transmodernidad como un proyecto de las periferias, la filósofa española Rodríguez Magda rechaza la visión "tercermundista" (15-16). Para ella la transmodernidad es el nuevo paradigma del primer mundo, globalizado, vacío, sofisticado, high tech. La definición de Rodríguez Magda incluye una nueva relación con la traducción. Mientras que la identidad "moderna" exalta las esencias, lo puro y homogéneo, siendo reacia a la traducción, esta se convierte en el modo constitutivo de las identidades transmodernas, híbridas y creolizadas de la era de la globalización. 
La Transmodernidad prolonga, continúa y transciende la Modernidad, es el retorno de algunas de sus líneas e ideas, acaso las más ingenuas, pero también las más universales. . . La Transmodernidad es el retorno, la copia, la pervivencia de una Modernidad débil, rebajada, light. La zona contemporánea transitada por todas las tendencias, los recuerdos, las posibilidades; transcendente y aparencial a la vez, voluntariamente sincrética en su "multicronía". La Transmodernidad es una ficción: nuestra realidad, la copia que suplanta al modelo, un eclecticismo canallesco y angélico a la vez (Rodríguez Magda 8).

A diferencia de la mirada sólida u opaca característica de la modernidad - es decir una mirada panóptica (Bentham) que vigila y disciplina (Foucault), o de la mirada fragmentada de la posmodernidad - la mirada "transmoderna" significa la capacidad de reunir, a distancia, transmisión y transparencia. Como escribe Rodríguez Magda, el panopticismo transmoderno "nos ofrece la hiperestesia de la mirada total, la transparencia indolora permanentemente presente desde la ausencia" (179). Paulatinamente, la "proliferación de la copia sobre la copia" conduce a una cultura de la copia sin original (181182). No resulta difícil reconocer en esta larga cadena de copias sobre copias el proceso de transculturaciones sucesivas; la cadena de hibridaciones, en donde cada original se convierte en copia que a su vez actúa como original de futuras copias. Original y copia se hacen intercambiables y terminan por confundirse. Nos encontramos aquí ante una teoría de la traducción que rompe radicalmente con la visión moderno-occidental en la que el original está rodeado de un aura de sacralidad.

\section{La traducción cultural desde una mirada latinoamericana}

La toma de conciencia de que Europa es hoy el escenario de procesos de transculturación tiene consecuencias epistemológicas importantes y requiere un cambio de perspectiva para esta región del mundo que, junto con el desarrollo de la modernidad, aprendió a verse como "punto cero" del conocimiento (Grosfoguel). Precisamente, al haberse construido o imaginado América Latina como la "otra cara" de Europa (de acuerdo con el binomio moderno/colonial), la inversión de la mirada - desde América Latina hacia Europa-constituye el eje a partir del que Europa puede entenderse a sí misma, ya no como un mosaico de culturas originales, homogéneas o puras, sino como resultado de procesos de transculturación, mestizajes y sincretismos.

Con una buena dosis de escepticismo, Ramón Grosfoguel se pregunta si existe un pensamiento "de-colonial" independiente del paradigma episte- 
mológico occidental dominante. Un inicio de respuesta se encuentra en las aportaciones latinoamericanas a la teoría de la traducción; aportaciones que se centran en la inversión de los paradigmas europeos. Cabe mencionar aquí el enfoque antropófago derivado del movimiento modernista de Oswald de Andrade (Brasil) o el enfoque subversivo representado por el argentino Jorge Luis Borges. En ambos casos se invierte la jerarquía original/copia y se exaltan las supuestas "imperfecciones" o "contaminaciones" de la copia hasta el punto de que el original es "engullido" por la copia (De Andrade, Manifesto Antropófago) y desaparece de nuestra mirada, o se hace totalmente superfluo ya que el conocimiento de la realidad (el original) se construye a partir de las conexiones entre las copias (confróntese con Borges, "Las versiones homéricas"). Estas visiones alternativas de la traducción son útiles para estudiar los procesos de hibridación y transculturación en una Europa "transmoderna" en la que las entradas y salidas de la modernidad - propias, según García Canclini, de las culturas híbridas-, están sacudiendo nuestros tradicionales esquemas de análisis social. El caso de Bruselas, capital de Europa, muestra una realidad - y más aun, una percepción generalizada - de que la frontera entre original y copia se va borrando en los procesos de transculturación.

Una mención especial merecen las reflexiones del antropólogo brasileño Eduardo Viveiros de Castro (2010), quien, a partir de la filosofía amerindia, desarrolló una teoría perspectivista de la traducción. Esta teoría, que parte de la coexistencia de una multiplicidad de perspectivas igualmente válidas, encierra una visión específica sobre la traducción cultural, no como posibilidad de "sustitución" basada en la semejanza metafórica, sino como "relación", basada en la contigüidad metonímica. El núcleo de esta teoría es el concepto de "equívoco"; de contigüidad en lugar de semejanza:

Contrariamente a esas patologías contingentes, el equívoco es una categoría propiamente trascendental, una dimensión constitutiva del proyecto de traducción cultural propio de la disciplina [antropológica]. Traducir es instalarse en el espacio del equívoco y habitarlo. No para deshacerlo, porque eso supondría que nunca ha existido, sino todo lo contrario, para destacarlo o potenciarlo, es decir, para abrir y ensanchar el espacio que imaginábamos que no existía entre los "lenguajes" en contacto, espacio ocultado justamente por el equívoco. El equívoco no es lo que impide la relación, sino lo que la funda y la impulsa: una diferencia de perspectiva. Traducir es presumir que hay, desde siempre y para siempre, un equívoco; es comunicar por la diferencia, en lugar de guardar al Otro en silencio presumiendo una univocalidad originaria 
y una redundancia última - una semejanza esencial— entre lo que él era y lo que nosotros "estábamos diciendo" (Viveiros de Castro 76).

Viveiros de Castro critica la definición de la antropología como "traducción cultural" y se pregunta: "¿Qué pasa cuando preguntamos a los indígenas qué es la antropología?"; en otras palabras, “¿[q]ué pasa cuando el clasificado se vuelve clasificador?" (69). Esta crítica la lleva a "invertir la inversión" del paradigma occidental a la que habían concluido los antropólogos de la llamada reverse anthropology. De acuerdo con Viveiros de Castro, "[el] concepto de perspectivismo propone una inversión de esa inversión: ahora le toca el turno al indígena, es the turn of the native, no the return of the native, como decía Adam Kuper" (74). Si antes hemos citado a Rodríguez Magda en cuya definición de la transmodernidad se habla del "retorno de la copia", para Viveiros de Castro no se trataría del retorno sino del turno de la copia. Teniendo en cuenta la realidad diversa de Europa hoy, el perspectivismo constituye, pues, una propuesta altercognitivista interesante.

\section{La tarea de la traducción en la Europa transmoderna}

Las cifras de Bruselas y Bélgica son ilustrativas de los profundos cambios demográficos registrados en Europa desde la Segunda Guerra Mundial a raíz de las migraciones económicas y la globalización. En la Europa transmoderna del siglo XXI, que es percibida por no pocos como "un mundo al revés", la tarea de la traducción se impone.

Citemos algunos ejemplos. En Francia hubo el caso, en 2010, de un argelino-francés que tenía cuatro mujeres; cada una de ellas recibía un subsidio social del Estado como madre soltera. Cuando el Ministro de Interior amenazó con quitarle la nacionalidad francesa por el delito de "poligamia", el imputado alegó que tenía cuatro "amantes": una práctica no tipificada como delito bajo la ley francesa. En abril de 2013, Francia aprobó, en medio de protestas masivas, la ley de matrimonio homosexual conocida como "ley del matrimonio para todos" (Loi du marriage pour tous). Aunque las protestas procedían de sectores católicos conservadores, algunos periodistas se preguntaban si en nombre de la igualdad republicana habría que incluir también en el futuro la opción musulmana de la poligamia. De la misma índole es el debate en torno al concepto de "acoso sexual". Mientras que Bélgica tipificó este delito en 2002 (ley del 11 de junio de 2002), Francia, donde el harcèlement (sexuel) había sido introducido en el código penal en 1992, decidió abrogar la ley en mayo de 2012 por falta de precisión en la definición de "los elementos constitutivos del delito". Para parte de la población musulmana, la ley que prohíbe el 
burka en los espacios públicos de Francia o de Bélgica es interpretada como una limitación a la libertad de religión o de expresión y, por tanto, como una incongruencia con los principios básicos de las democracias europeas. ¿Cómo establecer un consenso en sociedades en donde confluyen tradiciones culturales y religiosas muy diferentes y en donde, como hemos visto, gran parte de la población no participa de los procesos políticos? En un entorno multicultural, los conceptos se hacen resbaladizos y de contornos inciertos. ¿Qué significa hoy en Bruselas - o en cualquier metrópoli europea - el concepto de "emancipación" o de "religión"? ¿Cómo entender el derecho a la libertad religiosa? $\mathrm{Y}_{\mathrm{C}}$ cómo se interrelaciona este derecho con la igualdad de la mujer? ${ }_{\mathrm{C}} \mathrm{O}$ cómo se relaciona el derecho a la libertad de expresión y libertad de prensa con la libertad religiosa o el racismo? Estas cuestiones de difícil solución ocupan el debate público en la Europa de inicios del siglo XXI. La nueva realidad social rebasa las posibilidades de la traducción metafórica basada en la semejanza, y requiere un cambio de perspectiva de acuerdo con el molde perspectivista en el que la traducción se basa en la contigüidad. O, como explica un chamán amerindio, "con palabras usuales, me aplastaría sobre las cosas; con palabras torcidas, las rodeo y las veo claramente" (citado en Ventura i Oller 214). Sin adoptar el molde perspectivista de la traducción, la Europa transmoderna de hoy se aplasta sobre las cosas. El sistema semiótico de comunicación compuesto por un conjunto limitado de normas y signos mutuamente reconocidos está quebrado, y Europa se encuentra en un momento de transición, en el que los paisajes conceptuales tradicionales (ideoscapes, en terminología de Appadurai) están bajo tensión. Los modelos culturales, políticos y sociales que por el imperialismo y el colonialismo europeo se exportaron al mundo, están retornando hoy a Europa de forma transculturada, resemantizada a través del contacto con nuevos contextos durante su periplo migratorio. El gran desafío es cómo garantizar hoy en Europa un marco semiótico suficientemente estable y compartido que permita la comunicación intercultural en un paisaje humano cada vez más diverso e inestable.

El precedente de la primera globalización puede servir aquí de referente histórico. La llegada de los europeos (españoles y portugueses) al continente americano a finales del siglo XV significó la introducción de un sistema de signos a un entorno nuevo. Desde las primeras fechas de la llegada de los europeos al llamado Nuevo Mundo existen testimonios sobre el conflicto semiótico que este encuentro generó, denominado por Mignolo "semiosis colonial". Recordamos aquí que el concepto de "semiosis colonial" remite a un proceso relacional e interaccionista de producción de significados: 
Colonial semiosis is the general term to indicate a network of semiotic processes in which signs from different cultural systems interact in the productions and interpretation of hybrid cultural artifacts. In colonial semiosis the meaning of a sign no longer depends on its original cultural context (for instance, Castilian, or Amerindian, or Chinese), but on the new set of relations generated by communicative interactions across cultural boundaries. (Mignolo 262)

Esta producción de nuevos significados en el contexto de la colonización del continente americano fue el resultado de un proceso de traducción, entendida como creación de relaciones basadas en la contigüidad metonímica de elementos dispares. La traducción es vista aquí como un proceso de interrelacionamiento entre lenguajes semióticos distantes.

De acuerdo con Mignolo, la semiosis colonial se caracteriza por su dimensión híbrida, que resulta del traslado del signo (originado en un contexto geográfico y cultural determinado) a un contexto diferente (289). Desde una perspectiva histórica, el choque semiótico fue el motor de la transculturación de conceptos europeos introducidos en la realidad distinta del Nuevo Mundo. Un ejemplo es el concepto de "casta", que de significar lo étnicamente puro en España, adoptó en América, pluralizándose, el significado de lo mestizo ("las castas"). Otro ejemplo es el de "criollo", palabra de etimología incierta aunque originalmente sin ninguna connotación de mestizaje. Hoy, la creolité evoca la idea de contacto e intercambio intenso entre culturas de distintas procedencias. En el curso de la migración y su introducción en un contexto colonial, el signo original procedente del centro de la modernidad europea con su ideal de lo puro y homogéneo, se convirtió en copia contaminada, impura, imperfecta o mestiza. Las migraciones del siglo XX y la globalización confrontan a Europa con las versiones transculturadas de su propia realidad.

Proponemos aquí el concepto de "semiosis de-colonial" para el reencuentro conflictivo en Europa del supuesto "original" (el signo original procedente del centro de la modernidad europea con su ideal de lo puro y homogéneo) y sus "copias" transculturadas (contaminadas, impuras, imperfectas o mestizas elaboradas en la situación colonial) que, desde las periferias, han retornado al centro. Como observó George Marcus en su estudio de 1998, la etnografía multisituada en/del sistema-mundo implica un mayor desafío para la traducción. En la Europa del siglo XXI, confrontada con una "semiosis de-colonial", el necesario esfuerzo de traducción consiste en relacionar o crear convergencias, homologías o equivalencias entre conceptos en movimiento a través de la negociación intercultural. Para Europa, 
esta situación aparece como una realidad nueva. Si los conflictos semióticos habían sido más bien un problema de las periferias, ahora se ubican en el seno de los países centrales, y lo que antes era un problema exótico se ha convertido en un problema doméstico.

\section{Repensar Europa en tiempos de crisis}

En esta sección final reflexionaremos brevemente, a partir de dos noticias publicadas en la prensa belga, sobre qué significa, en términos antropológicos, la Europa transmoderna en términos de "nación".

La primera noticia, con fecha del 28 de noviembre de 2012, nos informa sobre una investigación judicial que tuvo lugar en Amberes, centro mundial de la industria del diamante, acerca del fraude comercial con diamantes sintéticos. La representante del Antwerp World Diamond Centre explica que "a simple vista resulta imposible distinguir un diamante sintético de un diamante natural. El diamante sintético es un verdadero diamante. El brillo es el mismo". ${ }^{7}$ Y añade que si el consumidor sigue prefiriendo el diamante natural, esto tiene que ver con "la magia del producto, debido a su origen natural y creciente escasez". La noticia nos recuerda el texto de Walter Benjamin, "La obra de arte en la época de su reproductibilidad técnica" (1936), en el que se explica que la reproductibilidad técnica de una obra de arte "atrofia" su aura, ya que el aura está asociada con la singularidad de su presencia dentro de una tradición vinculada a un tiempo y un lugar. La pérdida del aura cambia la función misma del arte, introduciéndolo en el ámbito de la política. Parafraseando a Benjamin, un concepto de "nación" en la era de la globalización coincide con una nación que ha perdido "su aura" y en donde la distinción entre original y copia ha perdido su funcionalidad. Por consiguiente, es un concepto transculturado de nación que se distancia tanto de la nación moderna (cultural o nacional) como de la nación premoderna (étnica o natural), y que pertenece ante todo al ámbito de la política. Para explicarlo nos referimos a la segunda noticia de la prensa belga.

En la segunda noticia publicada en noviembre de 2012, se hace referencia a "un hombre de 35 años, desempleado, natural de Molenbeek [un

7 La autora hace su propia traducción del fragmento del artículo a continuación transcrito: "Met het blote oog kan je het verschil niet zien", "Een synthetische diamant is een echte diamant, hij schittert op dezelfde manier als een natuurlijk diamant". "Zelfde schittering, ander gevoel". De Standaard. 28 de noviembre de 2012: 24-25. Impreso. 
barrio de Bruselas, C.S.], nacido en Bélgica, pero con raíces marroquíes". 8 De acuerdo con un concepto premoderno de nación, este hombre nacido en Bélgica probablemente podría ser definido como "amazigh" o bereber (su identidad étnica). Un concepto moderno de nación lo definiría como marroquí (su nacionalidad de origen), mientras que, de acuerdo con un concepto postmoderno de nación se podría definir como belga (su nacionalidad políticamente adquirida). En una visión transmoderna, sin embargo, resulta posible reconocer la realidad múltiple y migrante de la identidad que hoy ya es mayoritaria en Bruselas. En la capital de Europa, la nación ha perdido su aura. Bruselas nos confronta plenamente con la incómoda disyuntiva entre lo que vemos y lo que es. Los barrios con más apariencia extranjera - marroquí o turco, en general - resultan ser los más belgas en cuanto a la nacionalidad de sus habitantes, mientras que los barrios aparentemente más belgas (occidentales/europeos) son los de mayor porcentaje de extranjeros (muchos de ellos, funcionarios o empresarios procedentes de países europeos). Se da la contradicción de que mientras que el turco o marroquí está registrado como belga (y a pesar de su doble nacionalidad, Bélgica registra únicamente su nacionalidad belga), el europeo comunitario está registrado como extranjero en la propia capital de Europa. Resulta llamativo que el Estado transmoderno no registre la doble/múltiple nacionalidad de sus ciudadanos y que ni siquiera tenga los instrumentos para controlar las identidades transnacionales. Los métodos estadísticos de control demográfico, instrumento de la modernidad, fallan a la hora de catalogar esta "tercera nación" que ha emergido de la zona híbrida en la era de la globalización. Bruselas, capital de Europa y de Bélgica, es un espacio de traducción y mediación, en donde los conceptos están en movimiento, y los significados se han de negociar continuamente. Quedó abolida la frontera entre el original y la copia. La copia se ha convertido en auténtica. Bruselas nos presenta un espejo de lo que puede ser la Europa del siglo XXI.

\section{Concluyendo...}

Las grandes metrópolis europeas están inmersas en procesos de transculturación sin precedentes. La Europa del siglo XXI es hoy una tierra de frontera; un borderland en transformación que busca cómo desarrollar una "concien-

8 La autora hace su propia traducción del fragmento del artículo a continuación transcrito: "Redouane, een 35-jarige werkloze man uit Molenbeek, in "België geboren, maar met Marokkaanse roots". De Standaard. 29 de noviembre de 2012: 23. Impreso. 
cia de la mestiza", en palabras de la autora chicana Gloria Anzaldúa. En este contexto, hemos propuesto invertir la tradicional mirada eurocéntrica como estrategia epistemológica para el estudio de las nuevas realidades sociales y culturales europeas. Nuestro punto de partida ha sido la mirada que se organiza desde América Latina. Y hemos destacado el interés del enfoque TRANS para captar y entender la compleja realidad antropológica de un mundo globalizado. Es la perspectiva TRANS la que permite relacionar múltiples elementos distintos y distantes, fijando la mirada en el propio relacionamiento en lugar de las partes relacionadas.

Nuestras reflexiones se han desarrollado en torno a tres conceptos: transculturación, transmodernidad y traducción. En cuanto a esta última, hemos propuesto repensar las teorías eurocéntricas, privilegiando el paradigma perspectivista que, por su parte, permite deconstruir la dicotomía jerarquizada original/copia que define como sincretismos (impuros, inferiores) los productos de los procesos de transculturación. Quedó destacado que, de acuerdo con la definición de Ortiz, la transculturación es un proceso bi- o multidireccional del que nace una realidad nueva y original; un eslabón más en una cadena infinita de hibridaciones.

Observa Néstor García Canclini que ante una realidad multicultural se puede optar por vivir en "estado de guerra o en estado de hibridación" (20). Del éxito del proceso de transacciones (transculturación y traducción) propio de la hibridación dependerá, en gran parte, el futuro de la Europa transmoderna.

\section{Obras citadas}

Anzaldúa, Gloria. Borderlands / La Frontera. The New Mestiza.

San Francisco: Aunt Lute Books, 1987. Impreso.

Appadurai, Arjun. Modernity at Large. Cultural Dimensions of Globalization.

Minneapolis: University of Minnesota Press, 2008. Impreso.

Asad, Talal. "The concept of cultural translation in British Social Anthropology".

Writing Culture: The Poetics and Politics of Ethnography. Eds. James Clifford y

George E. Marcus. Berkeley: University of California Press, 1986. 141-164. Impreso.

"België geboren, maar met Marokkaanse roots". De Standaard.

29 de noviembre de 2012: 23. Impreso.

Bhabha, Homi K. The Location of Culture. London -

New York: Routledge, 1994. Impreso. 
Centeno, Miguel Ángel y Fernando López-Alves. The Other Mirror. Grand Theory Through the Lens of Latin America. Princeton: Princeton University Press, 2000. Impreso.

De Acosta, José. Historia Natural y Moral de las Indias. Edición crítica de Fermín del Pino-Díaz. Madrid: CSIC, 2008. Impreso.

Dussel, Enrique. "Transmodernidad e interculturalidad (Interpretación desde la Filosofía de la Liberación)". Enrique Dussel. Asociación de Filosofía y Liberación, 2004. Web. 13 de enero de 2015.

Fernández Retamar, Roberto. Calibán. Apuntes sobre la cultura en nuestra América. México: Diógenes, 1972. Impreso.

García Canclini, Néstor. Culturas híbridas: Estrategias para entrar y salir de la modernidad. Buenos Aires: Paidós, 2001. Impreso.

Grosfoguel, Ramón. "Transmodernity, border thinking, and global coloniality. Decolonizing political economy and postcolonial studies". Eurozine 1.23 (2008). Web. 13 de enero de 2015.

Koser, Khalid. International Migration. A Very Short Introduction. Oxford: Oxford University Press, 2007. Impreso.

Marcus, George E. Ethnography through Thick and Thin. Princeton: Princeton University Press, 1998. Impreso.

Mignolo, Walter. "The movable center: geographical discourses and territoriality during the expansion of the Spanish Empire". The Latin American Cultural Studies Reader. Eds. Ana del Sarto, Alicia Ríos y Abril Trigo. Durham: Duke University Press, 2004. 262-29o. Impreso.

Ortiz, Fernando. Contrapunteo cubano del tabaco y el azúcar. Madrid: Cátedra, 2002. Impreso.

Otte, Enrique, ed. Cartas privadas de migrantes a Indias, 1540-1616. México: Fondo de Cultura Económica, 1996. Impreso.

Poma de Ayala, Guaman. El primer nueva crónica y buen gobierno (1615). Web. 13 de enero de 2015.

Pratt, Mary Louise. Imperial Eyes. Travel Writing and Transculturation. London: Routledge, 1992. Impreso.

Rodríguez Magda, Rosa María. Transmodernidad. Barcelona: Anthropos, 2004. Impreso.

Saldívar, José David. Border Matters. Remapping American Cultural Studies. Berkeley - Los Angeles: University of California Press, 1997. Impreso.

Saldívar, José David. Trans-Americanity. Subaltern Modernities, Global Coloniality, and the Cultures of Greater Mexico. Durham: Duke University Press, 2011. Impreso.

Taylor, Diana. "Transculturating transculturation". Performing Arts fournal. 13.2 (2012): 90-104. Impreso. 
Ventura i Oller, Montserrat. "Chamanes amerindios: mediadores y traductores". Intelectuales, mediadores y antropólogos. La traducción y la reinterpretación de lo global en lo local. Eds. Mónica Martínez Mauri y Eugenia Rodríguez Blanco. San Sebastián - Donostia: Ankulegi Antropologia Elkartea, 2008. 207-220. Impreso.

Viveiros de Castro, Eduardo. Metafísicas caníbales. Líneas de antropología postestructural. Buenos Aires, Madrid: Katz, 2010. Impreso.

Zalamea, Fernando. "Peirce and Latin American 'razonabilidad': forerunners of transmodernity". European fournal of Pragmatism and American Philosophy. 1.1 (2009): 1-9. Web. 13 de enero de 2015.

"Zelfde schittering, ander gevoel". De Standaard. 28 de noviembre de 2012: 24-25. Impreso.

\section{Comentario de Vittoria Borsò}

(UNIVERSIDAD HEINRICH HEINE, DÜSSELDORF)

Christiane Stallaert propone un viraje total: ¡La dependencia es el problema del centro, no de la periferia! Este viraje es fruto de una transculturación entendida como desplazamiento del "locus" de la enunciación hacia América Latina. El reto es mirar a Europa desde América Latina; esto es: invertir la manera de ver el sistema mundial tradicional, para revisar, releer teorías clásicas europeas. ${ }^{9}$ ¡Este giro epistemológico es un desafío para las ciencias sociales europeas y sus marcos eurocéntricos! Se trata, pues, de luchar contra la colonialidad del saber frente a desigualdades de carácter multilateral, y de individuar mediaciones y mediadores frente a tal desafío. A la altura de las transformaciones que están ocurriendo en Europa tras los flujos de migraciones materiales e inmateriales - transformaciones que Stallaert demuestra con el ejemplo de Bruselas-, la Europa del siglo XXI "es hoy una tierra de frontera"; un borderland en transformación que puede aprovechar las experiencias del continente americano desde la "primera globalización" que acaeció con la llegada de los europeos.

Después del "post", Stallaert marca la necesidad de pensar el "trans" como (nuevo) paradigma de análisis de las culturas; un paradigma cuyas fórmulas creativas se encuentran en el contexto de la traducción ("transcultural" o transgressive translations) o de los Border Studies. Sin embargo, desde el

9 En este paradigma culminan mis reflexiones sobre la movilidad de la cultura. Confróntese por ejemplo "Pensar el movimiento: rutas e itinerarios de las culturas" de Borsò. 
comienzo, América Latina ha sido el laboratorio de una cultura basada en transferencias y transformaciones, y es en este sentido que Stallaert, con razón, rescata las propuestas de Fernando Ortiz o de Fernández Retamar. ${ }^{10}$

El itinerario de Stallaert consta de una revisión epistemológica de los conceptos afines al de transculturación, como el de la antropofagia de Oswaldo de Andrade, verificable en su escrito de 1938, o del más reciente concepto de hibridación de García Canclini, ${ }^{11}$ a su vez relacionado con la "infidelidad" de la traducción en culturas híbridas (de segunda mano), e inversamente asociado al concepto clásico ("traductor/traidor"), pues el proceso creador de la transposición es más importante que el resultado. Las "culturas híbridas" y la transculturación deniegan la fusión; subrayan el contrapunto. Stallaert formula entonces la importancia existencial e incluso ontológica de la traducción. La ontología que procede de esta función de la traducción es: "ser" quiere decir "ser en traducción" (being in translation). Después del giro textual ocurrido en la antropología con Writing Culture. The Poetics and Politics of Ethnography (1986) de Clifford y Marcus, en la actual fase de la globalización hacen falta epistemologías que correspondan a procesos multisituados, continuos e inacabables (Marcus, Ethnography Through Thick and Thin). Además, la traducción se está haciendo omnipresente también en las ciencias sociales, inspiradas por autores como Bonaventura de Sousa Santos o Homi Bhabha.

Frente a estos paisajes del siglo XXI, en los que se forman conexiones multilaterales, Stallaert se inclina, con razón, hacia una semiosis decolonial que confronta también a Europa con las versiones transculturadas de su propia realidad. Este concepto me parece acertado porque pone de relieve la transformación de la idea de "cultura original" que, en ambos lados del Océano, fundamenta los discursos eurocéntricos, ya deconstruidos por varios textos literarios de América Latina, entre otros, programáticamente, por Jorge Luis Borges.

10 Disiento de la tesis de Stallaert acerca de la prioridad de Mary Louise Pratt en recuperar la utilidad de la transculturación para las ciencias sociales. Pratt misma no reconoce todas sus deudas, por ejemplo con el concepto de "o-entre lugar" de Silviano Santiago. Mientras que Pratt relega a Santiago al contexto de suberalternity studies y a la modernidad de la periferia, el sociólogo brasileño, por el contrario, ya configura en los años setenta una epistemología del "trans". La importancia de Mary Louise Pratt reside tal vez en haber añadido el concepto de agency subrayando, como Malinowski y Fernando Ortiz, el proceso transitivo en ambas direcciones. Para todas estas argumentaciones confóntese "Entre Lugar" (Borsò)

11 La teoría de Canclini apareció en 1989, cinco años antes de la de Homi Bhabha, con la que tiene algunas premisas en común (por ejemplo el rol de la traducción). 
Ahora bien, Stallaert se relaciona con Walter Mignolo cuando subraya la calidad evidentemente híbrida de la semiosis colonial. No obstante, a mi modo de ver, la autora no toma en suficiente consideración que la "diferencia colonial" en la que se basa la semiosis colonial según Mignolo, sigue siendo "dependiente"; sigue oponiendo la "diferencia colonial" a la supuesta homogeneidad del centro europeo y a su episteme exclusivamente basada en separaciones. Dicha homogeneidad ha sido no solamente criticada anteriormente, sino que la misma Stallaert menciona los fallos del discurso del "occidentalismo" que borra tanto la ontología transcultural de las culturas, como todas las otras propuestas epistemológicas en la historia de la cultura de Europa y América (Borsò, "Pensar el movimiento: rutas e itinerarios de las culturas").

La relacionalidad en la que desembocan nuestras reflexiones, en respuesta al ensayo de Mabel Moraña (ver mi comentario al respecto), encuentra en el énfasis que Stallaert pone sobre la traducción un ulterior grado de dinámica. Se trata de echar luz sobre la trans-formación. "Trans" no es un puente, es decir una zona que relaciona diferencias, sino que es el movimiento de continua transcendencia de las diferencias - así se tendría que interpretar el concepto latino de trans-ferre: estar en las transferencias y mediaciones-. ${ }^{12}$ Aquí, el límite existe para ser transgredido. ${ }^{13}$ Llegamos por ende a una epistemología según la que la relación con el lugar no es dada, sino que está en continua formación, pasando, entre otras cosas, por la corporalidad. Justamente en esta capacidad reside, según Vilém Flusser, la "libertad del migrante" (Borsò, "Pensar el movimiento: rutas e itinerarios de las culturas"). Esta potencialidad, para la que se necesita una correspondiente política de la migración, es una figura existencial contraria a la anomia o la atopía de los migrantes en lugares de tránsito, entendiendo con estos conceptos la negación del derecho a un lugar dentro del nómos en el sentido de Carl Schmitt (unidad de localización y orden jurídico). ${ }^{14}$

Stallaert tiene razón cuando critica conceptos "transareales" que, según la "transmodernidad" propuesta por Dussel, encuentran en el diálogo transversal y simétrico entre periferias, como el diálogo Sur-Sur, una línea

12 Remito a la diferencia que propongo entre "Transitort" y "Transitorischer Ort" (lugar de tránsito vs. lugar de movimiento continuo, en el sentido mental y epistemológico). Confrontar con "Entre Lugar" de Borsò.

13 Entiendo "transcendente" en el sentido de Laclau, esto es: no como transcendentalidad plena, sino con una frontera demarcatoria nítida con respecto a lo empírico (Laclau, "Atisbando el futuro").

14 Semejante anomia y atopía es más bien el resultado de procesos de destructuración, como los que trata Pérez Brignoli en esta sección monográfica con el ejemplo de los moriscos. 
de fuga del occidentalismo supuestamente dominante en las relaciones transatlánticas. ${ }^{15}$ Más bien vale la pena considerar el rechazo del "tercermundismo" que la filósofa española Rodríguez Magda propone con su propio concepto de transmodernidad, referido al primer mundo globalizado que ahora parece haber superado "la identidad "moderna" que "exalta las esencias, lo puro y homogéneo". Ahora bien, otra vez, la transmodernidad de Rodríguez Magda, por la que opta Stallaert, se califica frente a la supuesta homogeneidad de la modernidad occidental; esto es: frente a un discurso cuyo poder sobre la cultura ofusca y excluye en Europa (y en América Latina) todas sus versiones alternativas o contestatarias. ¿Por qué limitarnos a este discurso? A mi modo de ver, nos ayudaría más la genealogía de las latencias que brotan del potencial de textos históricos en los que ya emergen agencialidades abiertas a una epistemología del movimiento -latencias invisibles en los discursos oficiales-. ${ }^{16}$

Que, a la altura de una epistemología "trans", América Latina sigue ofreciendo impulsos para repensar nuestras premisas, lo demuestra la teoría de la traducción del antropólogo brasileño Eduardo Viveiros de Castro desarrollada en 2010; teoría mencionada por Stallaert y basada en la "relación" metonímica, en lugar del criterio metafórico de la semejanza que da base a las teorías clásicas de la traducción: fórmula que revaloriza el equívoco como un agente activo que impulsa a ponerse en relación. ${ }^{17}$ Además, debemos a Viveiros de Castro la sugerencia de la inversión del lugar epistemológico. Es el "turno de la copia". Es el turno del nuevo mundo. Mirando desde él a Europa, descubriremos su compleja realidad antropológica e histórica y podremos, por ende, abandonar el esencialismo de nuestros propios discursos.

15 Este discurso deja incontestadas dos dicotomías: 1) la existente entre periferia y centro, 2) la que hay entre relaciones simétricas y asimétricas. Al contrario, cabe analizar la dinámica de las transformaciones en las cadenas de las traducciones y transferencias de Europa a América Latina y a la inversa. Cabría además preguntar por la complejidad histórica, lingüística, social y cultural de los contextos del diálogo Sur-Sur para no caer en nuevos "orientalismos" con la ilusión de escapar a la dependencia del "occidentalismo".

16 Acerca de un estudio genealógico de las latencias de lo posible en la historia de Europa remito a " $L a$ tency and the possible - Latency of the possible", congreso internacional que organicé con Christiane Voss y Lorenz Engell en el IKKM (Colegio Internacional para la Investigación de las Tecnologías de la Cultura y la Filosofía de los Medios), que tuvo lugar en Weimar el 7 y 8 de diciembre de 2014.

17 Ver la discusión sobre el Inca Garcilaso de la Vega en mi comentario a Pérez Brignoli. 


\section{Obras citadas}

Borsò, Vittoria. "Pensar el movimiento: rutas e itinerarios de las culturas".

México: migraciones culturales - topografías transatlánticas. Acercamiento a las culturas desde el movimiento. Eds. Vittoria Borsò, Yasmin Temelli y Karolin Viseneber. Frankfurt: Vervuert, 2012. 47-76. Impreso.

Borsò, Vittoria. "Entre Lugar". Iberoamerican postcolonialities.

Ed. Alberto Moreiras. En prensa. 2015. Impreso.

Laclau, Ernesto. "Atisbando el futuro". Laclau. Aproximaciones críticas a su obra. Eds. Simon Critchley, Oliver Marchart. Buenos Aires: Fondo de Cultura Económica, 2008. 382-383. Impreso. 